\title{
Fiber-Multi-Object Spectrograph (FMOS) for Subaru Telescope
}

\author{
Toshinori Maihara $^{a}$, Kouji Ohta $^{a}$, Naoyuki Tamura $^{a}$, Hiroshi Ohtani $^{a}$, Masayuki Akiyama $^{b}$, \\ Jun'ichi Noumaru ${ }^{b}$, Norio Kaifu ${ }^{b}$, Hiroshi Karoji ${ }^{c}$, Masanori Iye $^{c}$, Gavin B. Dalton ${ }^{d}$, \\ Ian R. Parry ${ }^{e}$, \\ David Robertson $^{f}$, Ray Sharples $f$, Deqing Ren ${ }^{f}$, Jeremy Allington-Smith ${ }^{f}$, \\ Keith Taylor ${ }^{g}$, and Peter Gillingham ${ }^{g}$ \\ ${ }^{a}$ Kyoto University, Kyoto 606-8502, Japan, \\ ${ }^{b}$ Subaru Telescope, NAO-J, Hilo, Hawaii, USA, \\ ${ }^{c}$ National Astronomical Observatory, Mitaka 181-8522, Japan, \\ ${ }^{d}$ University of Oxford, UK, \\ ${ }^{e}$ University of Cambridge, UK, \\ ${ }^{f}$ University of Durham, UK, \\ ${ }^{g}$ Anglo-Australian Observatory, Epping, Australia
}

\begin{abstract}
Design concept of the Fiber Multi-Object Spectrograph (FMOS) for Subaru Telescope together with innovative ideas of optical and structural components is presented. Main features are ; i) wide field coverage of 30 arcmin in diameter, ii) 400 target multiplicity, iii) 0.9 to $1.8 \mu \mathrm{m}$ near-infrared wavelengths, and iv) OH-airglow suppression capability. The instrument is proposed to be built under the Japan-UK-Australia international collaboration scheme.
\end{abstract}

Keywords: Fiber Spectrograph, Multi-object Spectrograph, Subaru Telescope, Prime Focus

\section{SCIENTIFIC OBJECTIVES}

The FMOS with capabilities of 400 multiplicity and $\mathrm{OH}$ suppression at near-infrared (NIR) wavelengths is expected to explore many scientific frontiers from nearby substellar objects to large scale structure of the high redshift universe. Major scientific targets to be tackled by spectroscopic surveys with FMOS are briefly described as follows:

Brown Dwarfs Spatial and mass distribution of the lowest mass stars and brown dwarfs will give clues for the understanding of initial mass function (IMF) and dark matter in the Galaxy. Nelson et al. (1993) predicted that there are more than 200 field brown dwarfs per square degree to a limiting magnitude of $H=22.5$ mag. NIR spectra of these objects at high resolution $(\mathrm{R}=1500 \sim 3000)$ can be used for detailed spectral classification and for accurate determinations of metallicity and temperature.

Protostars and Young Stellar Objects (YSOs) In order to investigate processes and physical mechanisms of star formation, infrared observations of highly obscured objects in molecular clouds are essential. Even without intracloud absorption, a majority of YSO's spectra intrinsically peaks in the $H$ band, and has large $\mathrm{Pa} \beta$ / $\mathrm{Br} \gamma$ line ratios, so that physical information can be recovered through $J$ and $H$ band spectroscopy. Studies of initial mass function (IMF) in star forming regions is also an important scientific objective with FMOS

Further author information: (Send correspondence to T.M.)

T.M.: E-mail: maihara@kusastro.kyoto-u.ac.jp 


\begin{tabular}{cccc}
\hline Scientific Area & Spectral Resolution & $\begin{array}{c}\mathrm{N} \\
\text { N/field }\end{array}$ & $\begin{array}{c}\text { magnitude } \\
\text { range }\end{array}$ \\
\hline \hline Brown Dwarfs & High & $>200$ & $H=22$ \\
Young Stellar Objects & High & $\sim 100$ & $H<19.5$ \\
Galactic Center Region & High & $>300$ & $H=18$ \\
LSS/Galaxy Evolution & Low & $>400$ & $H=22$ \\
AGNs/QSOs surveys & Low & $\sim 200$ & $H=22$ \\
\hline \hline
\end{tabular}

Table 1. Parameters for some of the key scientific goals for FMOS

Galactic Center Region The NIR coverage of FMOS allows the study of the kinematics and metallicity of stellar populations in the vicinity of the Galactic center, and the wide field of view allows current studies of the Milky Way's halo to be extended to more distant components at fainter limiting magnitudes. These data should yield powerful insights for star formation histories and formation process of the Milky Way galaxy as well as the Local Group galaxies.

Evolution of Galaxies and Large-Scale Structure atHigh Redshifts A next step for studying galaxy evolution and formation with $8-\mathrm{m}$ class telescopes is to probe activities of star formation in a quantitative way at redshifts $z>1$. At the epoch of such large redshifts, many optical lines serving as vital indicators of star formation history would be redshifted to NIR bands. In this context, multi-object spectroscopic observations with enhanced sensitivity through the $\mathrm{OH}$ suppression would play an important role, together with capabilities of medium resolution (a resolving power of about 1000) and of a wide wavelength coverage. The spectroscopy of extremely red objects (EROs) aiming at revealing their intriguing nature is also suitable for FMOS.

A systematic work of large scale structure as well as the clustering property of galaxies at higher redshift regions $(z>1)$ could be conducted by exploiting the expected FMOS performance as described so far. The wide field of view of FMOS (30 arcmin corresponds to about $10 \mathrm{Mpc}$ at $z=2$ ) will allow us to study large volume $\left(>\sim 10^{7} \mathrm{Mpc}^{3}\right)$ at early epochs.

Cosmological Evolution of AGN/QSO Population X-ray surveys for AGNs/QSOs have just started with Chandra and XMM-Newton and will provide unbiased samples of AGNs/QSOs out to a redshift of about 5 or even larger. Multicolor surveys of high-z QSOs are also in progress or under planning. Spectroscopic follow-up observations in optical to NIR bands of these samples are indispensable in studying the nature of them, which in turn may give clues of understandings towards an obscured population of AGN/QSO and their evolution in the cosmological timescale. An expected surface number density of these sources matches well with the number of fibers of the FMOS. (Mushotzky et al. 2000)

Table 1 summarizes the objects which are supposed to be targets of FMOS.

\section{PRIME FOCUS UNIT AND FIBER POSITIONER}

\subsection{Prime Focus Unit (PFU) Assembly}

The central section supported by spiders of the top ring of Subaru Telescope, which we call PFU, comprises the base ring, the corrector lens system with its housing, the fiber positioner, and the focal plane imager for the fiber alignment purpose. A hexsapod unit connects the base ring and the inner instrument base flange, from which the corrector lens system and the positioner with FPI are mounted. The latter portion is fixed on the instrument rotator flange to compensate field rotation. The conceptual design sketch of PFU is shown in Figure 1. 


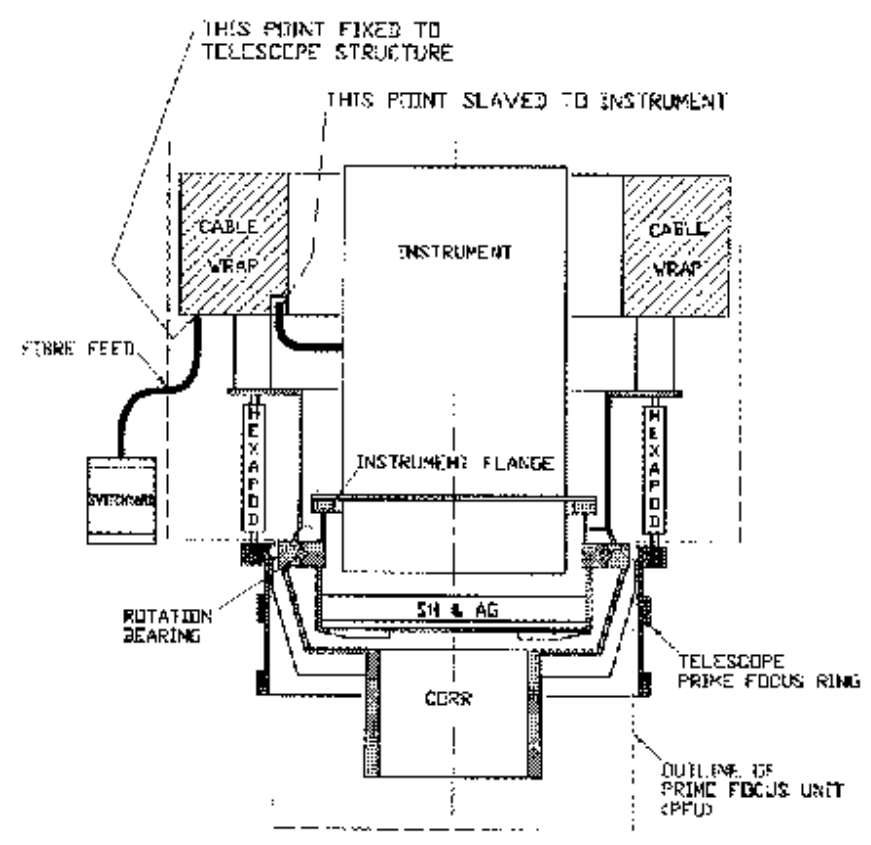

Figure 1. Cross sectional view of PFU. The shape of lenses of the corrector is not realistic.

\subsection{Prime Focus Corrector}

Since the corrector system currently used in the prime focus of Subaru Telescope is not intended to be used in the NIR region at longer wavelengths than $1 \mu \mathrm{m}$, it is necessary to have another corrector optimized in the 0.9 to $1.8 \mu \mathrm{m}$ region, and hopefully usable down to about $0.5 \mu \mathrm{m}$ for optical use. The specification of image quality of $0.5^{\prime \prime}$ diameter as an $80 \%$ energy circle is determined basically from the requirement of the fiber aperture of $1^{\prime \prime}$ in the MOS mode.

The result of optical design of the prime focus corrector with an inbuilt atmospheric dispersion correction (ADC) is schematically shown in Figure 2. Since the glass material of S-FPL52 will be difficult to make in large pieces, we "split-off" a low-powered "corrector plate" from the middle lens. The plate is positioned just behind the middle lens in such a way that its leading surface contacts the trailing surface of the middle lens at a stipulated minimum aperture determined by the marginal ray heights. The layout of the system is shown in Figure 2 with spot diagrams in Figure 3.

\subsection{Positioner and Focal Plane Imager}

Since the constraint of available space in the focal plane area of prime focus (about $145 \mathrm{~mm}$ in diameter) does not allow a conventional arrangement of positioner employed by AAT, for example, where the 400 "magnetic buttons" are deployed in the area of about $300 \mathrm{~mm}$ in diameter, we have to invent an innovative fiber positioning system in the smaller area in order to secure observability of the same number of objects. Recently, we have successfully developed a fiber positioning system based on impact-driven tube piezo-actuators. The result is separately reported in this conference by Gilligham et al. (2000). The positioner components (which we call "spines") are basically maneuvered by electric pulse trains of a saw-tooth shape, and actual positions are monitored by a focal plane imager CCD system so as to confirm the organized fiber positions. The monitor system allows us to give 


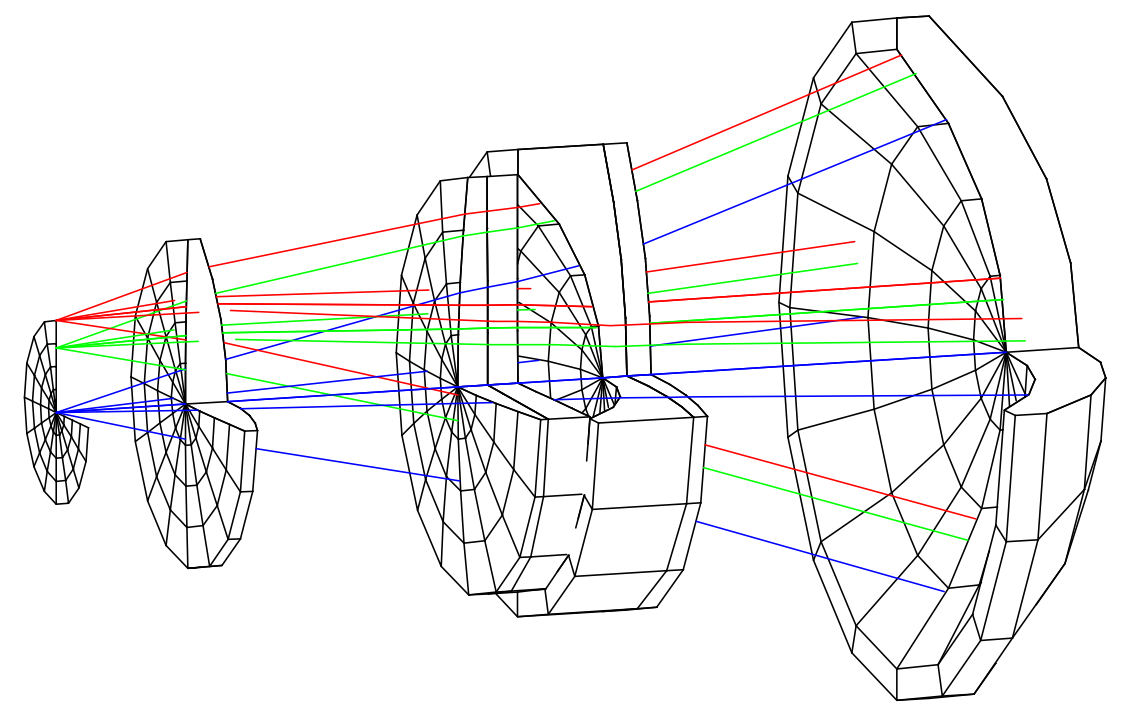

Figure 2. The schematic diagram of the prime focus corrector optics. This is a 4-element version in which an ADC is incorporated.

15 arcmin

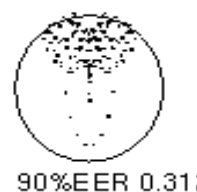

90\%EER 0.312

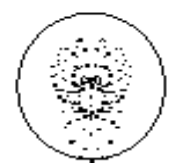
$90 \%$ EER 0.334

10.4 arcmin

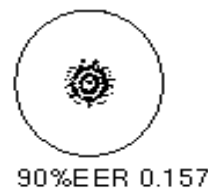

$400 \mathrm{~nm}$

Axial

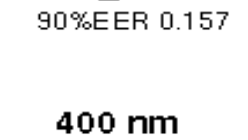

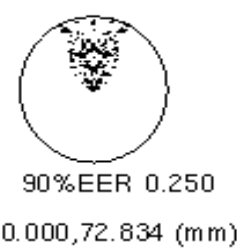

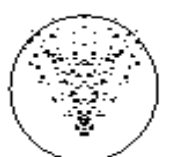

$90 \%$ EER 0.401
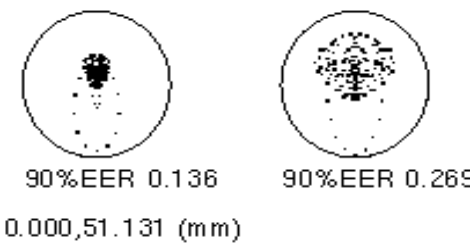

$90 \%$ EER 0.269

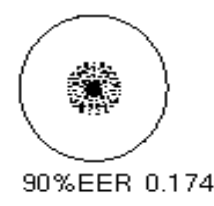

$0.000,0.000(\mathrm{~m} \mathrm{~m})$

$690 \mathrm{~nm}$

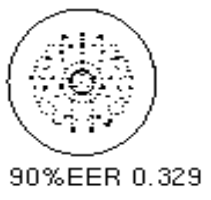

$1800 \mathrm{~nm}$

Circle is 1 arcsec in diameter, EER units are arcsec

Figure 3. The spot diagram of the 4-element prime focus corrector. 


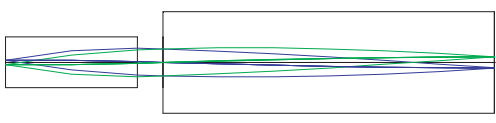

(a)

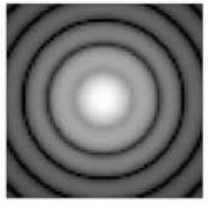

(b)

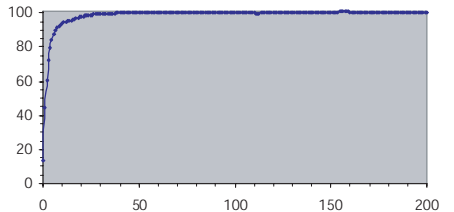

(c)

Figure 4. The fiber-fiber connector: (a) Optical layout. The collimating (left) and focusing (right) lenses are Selfoc SLW1.0 and Selfoc SLS2.0 gradient index microlenses, respectively; (b) Simulated Point Spread Function (PSF) of the Selfoc SLW1.0 collimating microlens. The PSF is diffraction limited in the 0.9-1.8 mm wavelength range; (c) A measurement of encircled energy for the Selfoc SLW1.0 collimating microlens. The horizontal axis is the radius in microns and the vertical scale is the fractional enclosed energy in percent.

information of positional errors, which can be corrected by one or two iterative operations of the actuator. (See the details presented by Gilligham et al. 2000).

\subsection{Fibers and Fiber Connectors}

The input focal ratio at the prime focus of Subaru Telescope is F/2 to which an NA of fibers has to be matched. Currently we assume that the fiber core-diameter of MOS mode is $100 \mu \mathrm{m}$ or $1.2^{\prime \prime}$ on the sky. ( $85 \mu \mathrm{m}$ fibers were also be considered, but it is found that available fibers made of silica-glass type favor a $100 \mu \mathrm{m}$ fiber in terms of the NA constraint.) Because the PFU must be removed from the telescope when FMOS is not in use, an optical fiber-fiber connector is used to provide a break in the longfiber feed at the top-end ring. Fiber-fiber connectors which convert from $\mathrm{F} / 2$ to $\mathrm{F} / 5$ are necessary to match the input focal ratio for the NIR spectrograph. In the present design we plan to build simple F-conversion couplers connecting the output $\mathrm{F} / 2$ beam to the input of second F/5 fibers. Dichroic couplers are envisaged as a future option for the simultaneous optical spectroscopy mode of FMOS. Each converting coupler comprises a pair of matched SELFOC gradient index lenses as a collimator and a focusing lens. The expected efficiency of coupling is estimated as $95 \%$ due to the aberration (about $3-4 \mu \mathrm{m}$ ) of the lens system over the wavelength range from 0.9 to $1.8 \mu \mathrm{m}$.

\section{OH-AIRGLOW SUPPRESSOR AND INFRARED SPECTROGRAPH}

\subsection{OH-Airglow Suppressor Optics}

Since the wavelength bands of FMOS suffer badly from OH-airglow lines, it is essential to eliminate as much $\mathrm{OH}$ lines as possible, while the advantage of the $\mathrm{OH}$ suppression effect being proven by the first-generation $\mathrm{OH}$ suppressor (e.g. Iwamuro et al.1996), and the second-generations are also planned to be built for $8 \mathrm{~m}$-class telescopes (Maihara et al. 2000, Parry et al. 2000). In the present FMOS design we have adopted the optical layout for $\mathrm{OH}$ suppression similar to CIRPASS (Cambridge IR Panoramic Survey Spectrograph) which has been proposed by Parry et al.(2000) as a fiber-based, integral field and multi-object spectroscopic instrument at Gemini's F/16 Cassegrain focus. It has a fast F-ratio, and is fairly compact. Nevertheless, the number of objects to be measured simultaneously of about 400 can only be handled by two identical spectrographs. The 


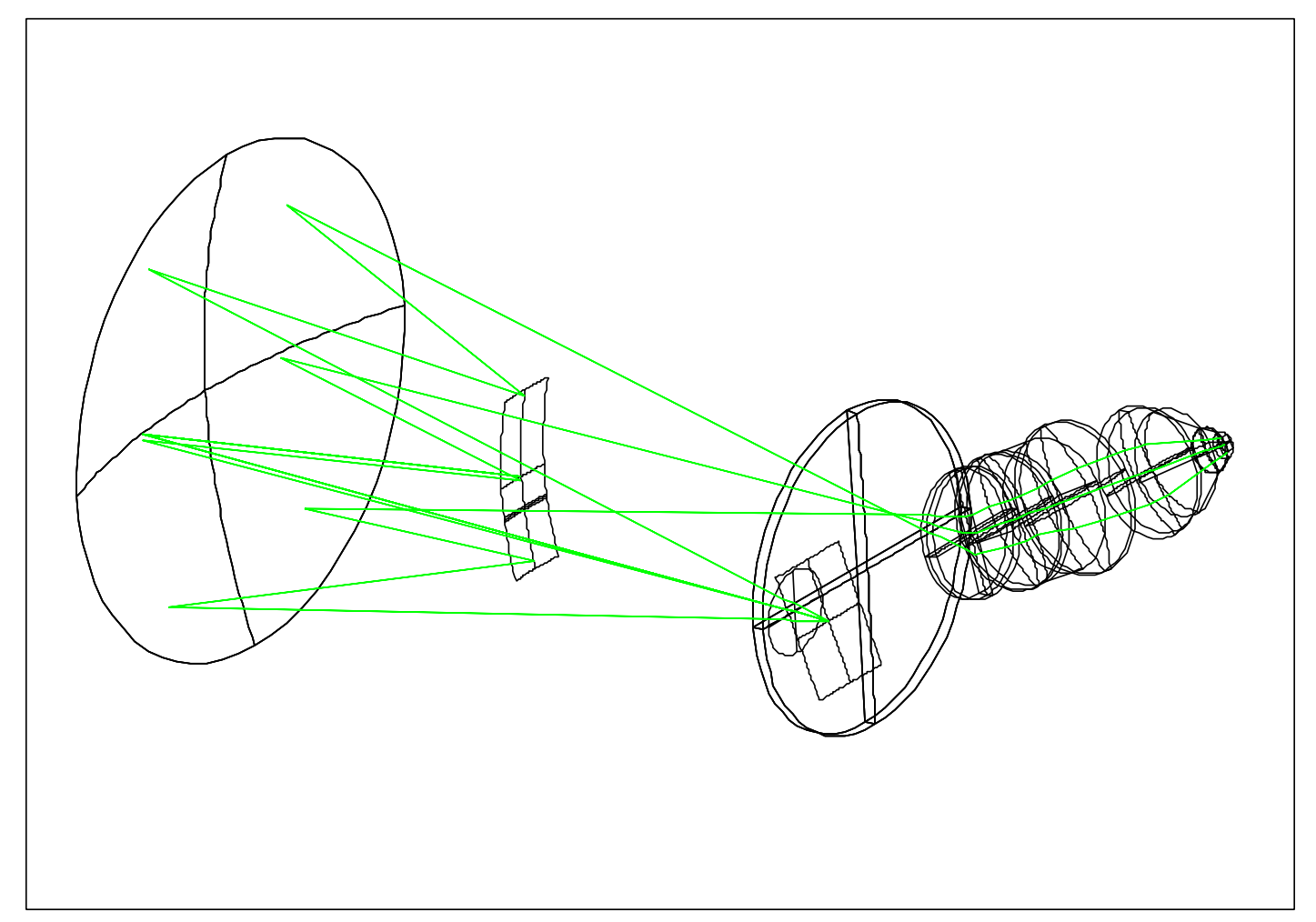

Figure 5. A 3D optical layout of the NIR spectrograph

conceptual layout of an NIR spectrograph optics is shown in Figure 5, and the specifications of each component are presented in Table 2 .

The beam train of the OH-airglow suppressor optics is schematically shown in Figure 6 (a) and (b), in which optical components are multiple times put on along the real optical path.

\subsection{Mask Mirror}

Masking of $\mathrm{OH}$ airglow lines is secured by line absorbers put on the so-called "Mask Mirror" where the first spectroscopic images are focused. The mirror is of concave sphere, concentric with the Offner mirror. In the center of the mirror of the size of $140 \mathrm{~mm} \times 460 \mathrm{~mm}$, a thin rectangular through-hole is to be excavated for putting a slit assembly. The masking plate is made of a thin metal by the photo-chemical etching method, so that about 200 absorbing lines are placed throughout the wavelength range of FMOS. A reduction of background of a factor of about 20 is expected in the $\mathrm{H}$ band, while the factor being somewhat lower in shorter spectral bands,

\subsection{Volume Phase Holographic Grating}

Considering recent research and development efforts of Volume Phase Holographic (VPH) grating (e.g., see report of NOAO: http://www.noao.edu/ets/vpgratings/), we have decided to utilize the VPH grating as an inversedispersive element, with which the outgoing light transmitting through the grating becomes a spectroscopically combined beam of a wider spectral range with a lowered resolution. This functional requirement of the FMOS NIR spectrograph meets with expected performance of VPH grating, although the fabrication technology has still been in progress.

Specifications of the VPH grating in the NIR spectrograph is summarized as, i) groove number of 340 lines $/ \mathrm{mm}$, ii) deflection angle of $27.9^{\circ}$, iii) ruled area of $210 \times 320 \mathrm{~mm}^{2}$, and the thickness of $20 \mathrm{~mm}$. With this configuration, the optical throughput is expected to be about $60 \%$ at the peak and about $40 \%$ in the shorter 

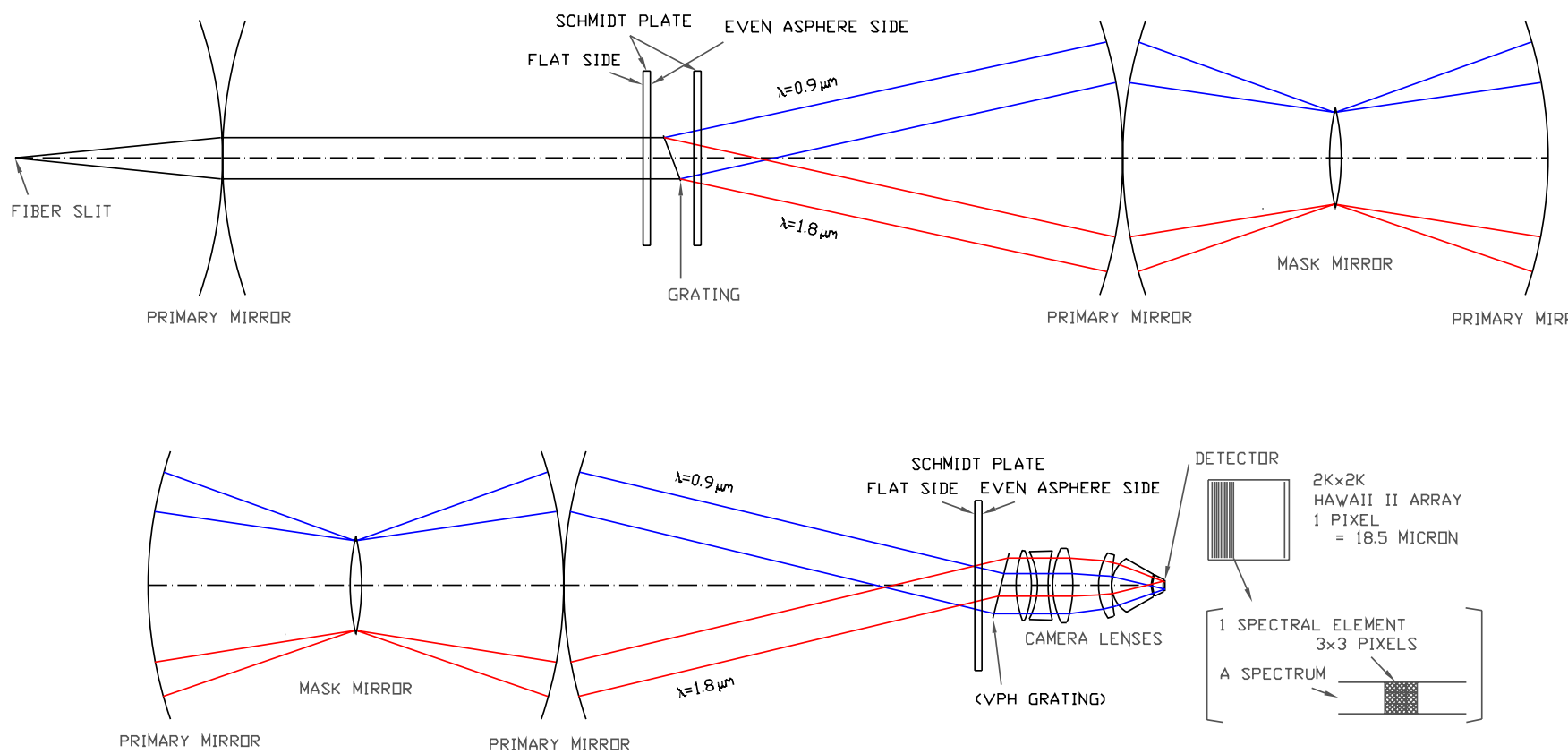

1000

Figure 6. The beam train presentation of the $\mathrm{OH}$ suppressor optics. Upper: Optical elements from the input slit to the big Offner mirror are schematically shown. Lower: The similar beam train presentation of the re-focusing big mirror to the detector, including the achromatic NIR camera is schematically shown. 


\begin{tabular}{|c|c|c|c|c|c|}
\hline No. & Component & Surface & $\begin{array}{c}\text { Curvature }^{a} \\
(\mathrm{~mm})\end{array}$ & $\begin{array}{c}\text { Optics Size } \\
(\mathrm{mm})\end{array}$ & $\begin{array}{c}\text { Beam Size } \\
(\mathrm{mm} \times \mathrm{mm})\end{array}$ \\
\hline$\overline{11}$ & $\overline{\text { Primary Mirror }}$ & Sphere & 1940 & $1260 \phi$ & 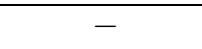 \\
\hline 2 & Schmidt Plate & Flat/Even ${ }^{b}$ & - & 800 & $209 \times 209$ \\
\hline 3 & $\begin{array}{c}\text { Grating } \\
\left(500 / \mathrm{mm}, \theta_{b}=20.74^{\circ}\right)\end{array}$ & Flat & - & $240 \times 310$ & $209 \times 223$ \\
\hline 4 & Schmidt Plate & Flat/Even & - & 800 & $209 \times 262$ \\
\hline 5 & Primary Mirror & Sphere & 1940 & $1260 \phi$ & - \\
\hline 6 & Mask Mirror & Sphere & 995 & $164 \times 460$ & - \\
\hline 7 & Primary Mirror & Sphere & 1940 & $1260 \phi$ & - \\
\hline 8 & Schmidt Plate & Flat/Even & - & 800 & $209 \times 262$ \\
\hline 9 & $\begin{array}{c}\text { VPH grating } \\
\left(\theta_{\text {deflection }}=27.9^{\circ}\right)^{c}\end{array}$ & Flat & - & $323 \phi$ & $209 \times 317$ \\
\hline
\end{tabular}

${ }^{a}$ An radius of curvature for each component is indicated.

$b$ "Even" means an even asphere.

${ }^{c}$ The angle by which the light axis deflects after passing VPH.

Table 2. IRS Optical Components

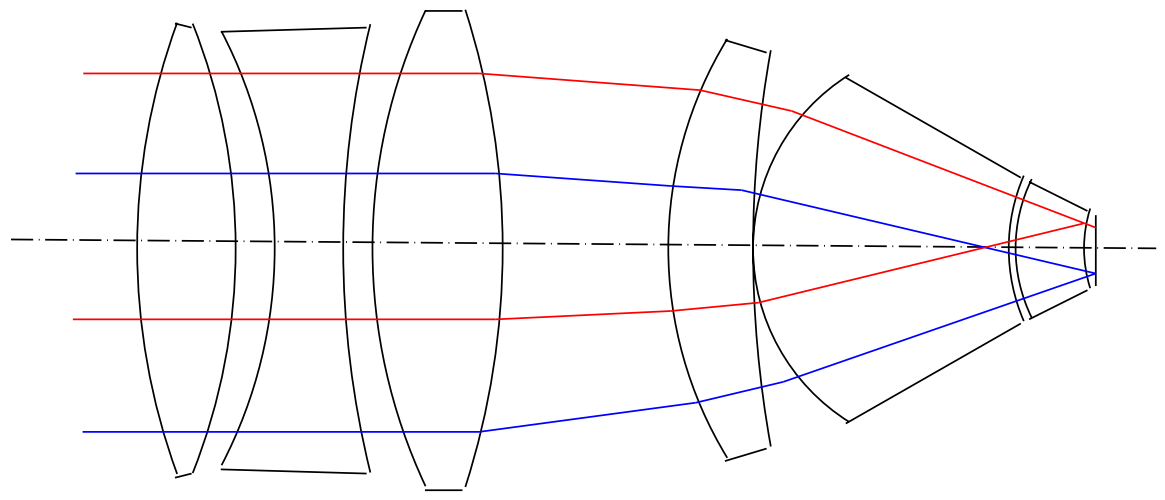

Figure 7. The cross sectional view of the achromatic NIR camera. The specifications of each lens are tablated in Table 3 .

and longer edges of the band. The resultant spectrum obtained at the focus of the F/1.2 spectrograph camera has a spectral resolving power of about 900 in average over the entire wavelength range.

\subsection{Infrared Camera and Detector}

The optical layout of an achromatic camera design is presented in Figure 7, and the components of optics are tabulated in Table 3. The incoming beam through the VPH grating of $\mathrm{F} / 5$ is re-imaged onto a $\mathrm{MCT} 2 \mathrm{~K} \times 2 \mathrm{~K}$ (HAWAII II) array, thereby realizing full-wavelength covered spectra of about 220 target objects. Since the sizes of optical elements are fairly large, feasibility of lens fabrication is not fully explored, especially large uniform crystals of $\mathrm{BaF}_{2}$ may be hard to obtain, not impossible though.

\section{SUMMARY}

The instrument concept of the planned fiber-based multiobject spectrograph (FMOS), a wide field-covered multiobject spectrometer utilizing the prime focus of Subaru Telescope is presented in connection with major scientific objectives together with the present status of developments. Design studies under the multinational collaboration 


\begin{tabular}{|c|c|c|c|c|c|c|}
\hline No. $^{a}$ & Component & Surface & Glass & $\begin{array}{c}\text { Curvature } \\
(\mathrm{mm})\end{array}$ & $\begin{array}{l}\text { Diameter } \\
(\mathrm{mm})\end{array}$ & $\begin{array}{c}\text { Beam Size } \\
(\mathrm{mm} \times \mathrm{mm})\end{array}$ \\
\hline \multirow[t]{2}{*}{10} & \multirow[t]{2}{*}{ Lens 1} & Sphere & \multirow[t]{2}{*}{$\overline{\mathrm{CAF}_{2}}$} & 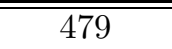 & 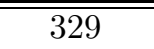 & $209 \times 324$ \\
\hline & & Sphere & & 444 & 328 & $202 \times 323$ \\
\hline \multirow[t]{2}{*}{11} & \multirow[t]{2}{*}{ Lens 2} & Sphere & \multirow[t]{2}{*}{ BAF51 } & 342 & 316 & $191 \times 311$ \\
\hline & & Sphere & & 685 & 327 & $191 \times 322$ \\
\hline \multirow[t]{2}{*}{12} & \multirow[t]{2}{*}{ Lens 3} & Sphere & \multirow[t]{2}{*}{$\mathrm{CAF}_{2}$} & 408 & 347 & $195 \times 341$ \\
\hline & & Sphere & & 569 & 348 & $192 \times 343$ \\
\hline \multirow[t]{2}{*}{13} & \multirow[t]{2}{*}{ Lens 4} & Sphere & \multirow[t]{2}{*}{$\mathrm{BAF}_{2}$} & 293 & 306 & $164 \times 302$ \\
\hline & & Sphere & & 814 & 289 & $147 \times 285$ \\
\hline \multirow[t]{2}{*}{14} & \multirow[t]{2}{*}{ Lens 5} & Sphere & \multirow[t]{2}{*}{$\mathrm{CAF}_{2}$} & 148 & 253 & $141 \times 251$ \\
\hline & & Sphere & & 134 & 106 & $41 \times 100$ \\
\hline \multirow[t]{2}{*}{15} & \multirow[t]{2}{*}{ Lens 6} & Sphere & \multirow[t]{2}{*}{ SF56A } & 114 & 101 & $37 \times 94$ \\
\hline & & Even & & 96 & 58 & $10 \times 48$ \\
\hline
\end{tabular}

${ }^{a}$ This number is continued from Table 1.

Table 3. IRS Optical Components

scheme show that unique features of FMOS, such as the multiplicity of measurements of about 400 targets within a 30 arcmin field, the simultaneous, full wavelength coverage from 0.9 to $1.8 \mu \mathrm{m}$, and the $\mathrm{OH}$-airglow suppression capability to gain high sensitivity, can be realized, although specifications of some critical components are still to be improved.

\section{ACKNOWLEDGMENTS}

Authors would like to acknowledge contributions of Richard Ellis, Alan Holmes, Ian Lewis, and Craig Mackay in promoting the FMOS project. Thanks are also due to Prof. Higuchi of University of Tokyo for precious advice in designing impact driven piezo actuators. The FMOS project in the definition phase of conceptual design has jointly been sponsored by NAO (Japan), PPARC (UK) and AAO.

\section{REFERENCES}

1. Iwamuro,F., Oya,S., Tsukamoto,H., and Maihara,T. 1996, ApJ, 466, L70-L73

2. Maihara, T., and Iwamuro, F. 2000 in Imaging the Universe in Three Dimensions: Astrophysics with Advanced Multi-Wavelength Imaging Devices (eds. W. van Breugel \& J. Bland-Hawthorn), ASP Conf. Series, in press

3. Mushotzky, R.F., Cowie, L.L., Barger, A.J., Arnaud, K.A. 2000, Astro-ph/0002313

4. Nelson, L.A., Rappaport, S., Joss, P.C. 1993, ApJ 404, 723

5. Parry, I.R., Dean, A.J., Ellis,R.S., King, D., Mackay, C.D., McMahon, R.G., Medlen, S.R., Pritchard, J.M., and Ramaprakash, A.N. 2000, in Imaging the Universe in Three Dimensions: Astrophysics with Advanced Multi-Wavelength Imaging Devices (eds. W. van Breugel \& J. Bland-Hawthorn), ASP Conf. Series, in press

6. Gillingham, P., Miziarski, S., Akiyama, M., and Klocke,V. 2000 Proc. SPIE 4008, in press 